\title{
Evaluation of the Value of Nursing Intervention Guidance in Controlling the Fatigue Level of Patients with Breast Cancer
}

\author{
PING. GONG, JIAN HONG. LIU AND XIAO RONG. ZHOU*
}

Department of Medical Oncology, Jiangsu Cancer Hospital, Jiangsu Institute of Cancer Research, Cancer Hospital Affiliated to Nanjing Medical University, No.42 Baiziting, Nanjing, Jiangsu 210009, China

Gong et al.: Nursing Intervention Guidance in Controlling the Fatigue Level of Patients with Breast Cancer

\begin{abstract}
To investigate the effect of the continuous nursing intervention model on postoperative fatigue levels and quality of life in breast cancer patients. A total of 112 patients with breast cancer who received radiotherapy and chemotherapy in the oncology department of our hospital from January 2018 to January 2020 were collected and randomly divided into the observation group $(n=56)$ and the control group $(n=56)$. The general clinical data of the two groups were comparable. Patients in the control group were instructed with conventional nursing interventions, while those in the observation group were given continuous nursing management in addition to conventional nursing care, and their mastery of nursing management health, quality of life and changes in cancer-related fatigue status were assessed by the selfadministered questionnaires, the European Organization for Research and Treatment of Cancer Quality of Life Questionnaire-Core 30, and the revised piper fatigue scale, respectively. The results showed that only $35(62.50 \%)$ patients in the control group were completely compliance, $4(7.14 \%)$ were partially compliance, and $17(30.36 \%)$ were non-compliance, while 48 (85.71\%) were completely compliance, $6(10.71 \%)$ were partially compliance, and $2(3.58 \%)$ were non-compliance in the observation group, the difference between the two groups was statistically significant $(p<0.05)$. After nursing, the quality of life scores of patients in the control group and the observation group were significantly improved, and the revised piper fatigue scale scores decreased significantly, the quality of life of the patients and the cancer related fatigue in the observation group was more significantly improved than that in the control group, and the difference was statistically significant $(\mathbf{p}<\mathbf{0 . 0 5})$. In the nursing intervention of breast cancer patients, the continuous intervention model can improve the life and mental state of breast cancer patients significantly, to improve their quality of life, alleviate the symptoms of cancer-related fatigue, and promote the rehabilitation of breast cancer patients.
\end{abstract}

Key words: Breast cancer; nursing intervention; cancer related fatigue; quality of life

Breast cancer was one of the most common cancers that threaten the women's health, which was caused by many factors, such as age, genetics, and alcoholism ${ }^{[1]}$. At present, the clinical treatment for patients with the middle-advanced breast cancer was surgery combined with chemotherapy. Although it can control the condition of the patients to a great extent, the side effects during chemotherapy bring great pain to the life and spirit of the patients ${ }^{[2]}$. It has been found that the breast cancer patients undergoing chemotherapy have fatigue experience, which will affect the patients' physiological, psychological, and cognitive functions in varying degrees, and then affect the patients' quality of life ${ }^{[3]}$. Although most oncologists recognize the benefits of care for breast cancer patients, many doctors believe that the patients lack the awareness and time of nursing management, as well as concerns about the lack of continuous nursing counseling during treatment $\mathrm{t}^{[3-5]}$. In this study, the continuous nursing intervention was adopted to manage the breast cancer patients, and the application value of nursing intervention in postoperative chemotherapy was analyzed. A total of 112 patients with breast cancer who received radiotherapy and chemotherapy in the oncology department of our hospital from January 2018 to January 2020 were collected and randomly divided into the observation group $(n=56)$ and control group $(n=56)$. The age of the observation group was 37 $-69 \mathrm{y}$ old, the average age of the observation group was $46.25 \pm 1.75 \mathrm{y}$ old, and the average course of disease was

*Address for correspondence 
$29.80 \pm 2.55 \mathrm{mo}, 37$ patients had a reproductive history, 19 patients had no reproductive history, 48 patients with unilateral breast cancer, 8 patients with bilateral breast cancer. The age of the control group was 36-69y old, the average age was $47.10 \pm 1.20 \mathrm{y}$ old, the average course of disease was $28.20 \pm 1.90 \mathrm{mo}, 34$ cases had a reproductive history, 22 cases had no reproductive history, 46 patients with unilateral breast cancer, and 10 patients with bilateral breast cancer. Statistical analysis showed that the clinical data of the two groups had no statistical significance (fig. 1) ( $p>0.05$ ). For the patients in the control group, some health knowledge education was used, guide patients on the diet, exercise, and medication. Patients were advised to take medicine on time, regulate diet, and exercise properly, and maintain an optimistic and cheerful attitude to face the disease, and come to the hospital regularly for re-examination. The patients in the observation group were followed up by telephone twice a $\mathrm{w}$ on the basis of the guidance of the patients in the control group. Make a reasonable diet plan; help the patients in medication and life to keep abreast of the patients' condition, life, and psychological changes. At the same time of telephone follow-up, patients were urged to watch some inspirational and healing film and television works. Enlighten patients to actively participate in some social activities and physical exercise to integrate into society. In addition, we should formulate some reasonable rehabilitation plans and instruct the patients to strengthen the rehabilitation training and enhance their immunity, and the rehabilitation training was limited to some simple rehabilitation activities. For some special patients, arrange the medical staff who participates in the study to provide detailed guidance and help to promote the recovery of patients. This study lasted for $3 \mathrm{mo}$. Scoring through telephone follow-up survey or patient re-examination, which mainly includes the following aspects: Compliance assessment: It can be divided into three categories, complete compliance (actively obeys the rehabilitation plan training or instructions made by the medical staff), partial compliance (obeys part of the rehabilitation plan or must be under the repeated supervision of the family members), and no compliance (does not implement the rehabilitation plan made by the medical staff).

Nursing management: To test the patients' mastery of nursing knowledge, we designed a questionnaire of health knowledge, including the healthy diet, functional exercise, myelosuppression, skin reaction, and basic chemotherapy $(5 * 20)$. The higher the score, the better the patients' knowledge of nursing management. Quality of life score: The European organization for Research and treatment of cancer quality of life Questionnairecore 30 (EORTCQLQ-30) was used to evaluate the quality of life of patients, including the family support, psychological activities, daily life, health perception, and activity ability (1-4 grades). The higher the grade, the higher the quality of life of the patients ${ }^{[6]}$. Cancer related fatigue: The cancer-related fatigue of breast cancer patients was evaluated by the revised piper fatigue scale (PFS-R), which mainly included the physical, cognitive, emotional, and behavioral scores. The scores ranged from 0 to 10 , which divided into no fatigue (0 points), mild fatigue (1-3 points), moderate fatigue (4-6 points), and severe fatigue ( $\geq 7$ points). The data were analyzed by SPSS 20.0. The measurement data were expressed by $\mathrm{x} \pm \mathrm{S}$ and analyzed by t-test; the counting data were expressed by percent and analyzed by the $\chi^{2}$ test. $\mathrm{p}<0.05$ indicates that the difference is statistically significant. The results showed that only $35(62.50 \%)$ patients in the control group complete compliance the rehabilitation plan, $4(7.14 \%)$ partial compliance and 17 (30.36\%) non-compliance. In the observation group, 48 cases $(85.71 \%)$ were in complete compliance, 6 cases $(10.71 \%)$ were in partial compliance, and 2 cases (3.58 \%) were non-compliant. The difference between the two groups was statistically significant $(p<0.05)$ (fig. 2). The results showed that the mastery of nursing management knowledge in

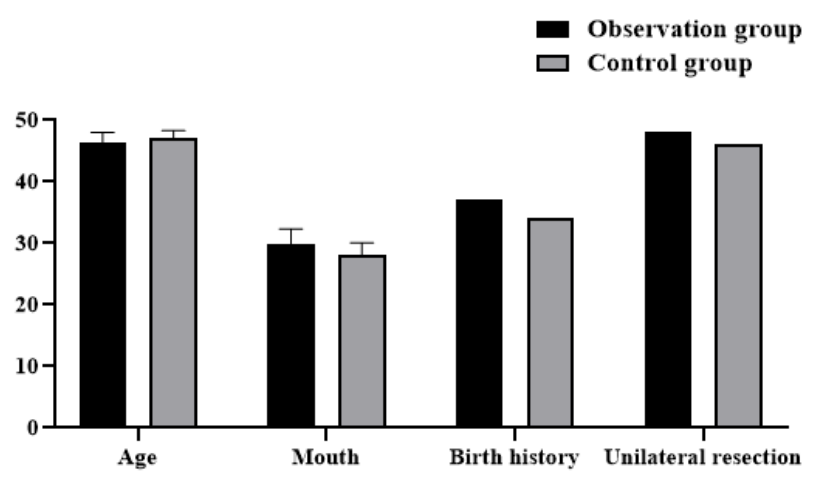

Fig. 1: Comparison of the clinical data between the two groups

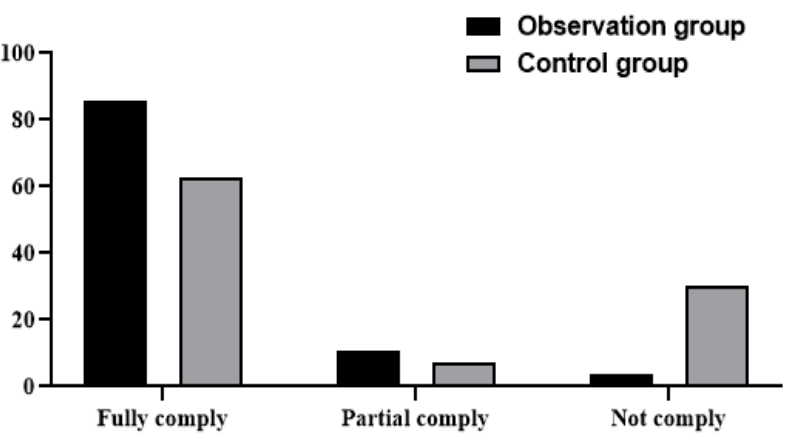

Fig. 2: Compliance evaluation 
the observation group was significantly better than that in the control group, and the difference between the two groups was statistically significant $(p<0.05)$ (fig. 3). The results showed that after nursing, the quality of life scores of patients in the control group and the observation group were significantly improved, and the quality of life of the patients ${ }^{[7]}$ in the observation group was more significantly improved than that in the control group, and the difference was statistically significant $(\mathrm{p}<0.05)$ (fig. 4$)$. The results showed that after nursing, the PFS-R scores of the patients in the control group and the observation group decreased significantly, and the PFS-R scores of the patients in the observation group decreased more significantly than those in the control group, and the difference between the two groups was statistically significant $(p<0.05)$ (fig. 5). Breast cancer was one of the most common tumors in women, and its pathogenic factors were complex, including alcoholism, postmenopausal obesity, late childbearing, and infertility ${ }^{[3,8-10]}$. With the development of society, the female has played a more important role in social development in recent y. And the pressure faced by the female has increased sharply, resulting in a significant increase in the incidence of breast cancer ${ }^{[11,12]}$. At present, there were no specific drugs for breast cancer, which was generally treated by surgery combined with postoperative radiotherapy and chemotherapy ${ }^{[13-16]}$. Although this treatment can help patients control the disease and prolong survival, postoperative radiotherapy and chemotherapy often cause nausea and vomiting, hair loss, and infection. At the same time, the physical disability caused by surgery and the physiological stress response caused by chemotherapy makes the patients depressed and depressed. Therefore, the implementation of nursing intervention for patients is an important measure to promote the patients' good recovery and improve their quality of life ${ }^{[17]}$. A continuous nursing intervention model was adopted in this study. The results showed that the scores of the family support, psychological activity, daily life, health perception, and activity ability in the EORTC quality of life questionnaire (QLQ)30 scale of the observation group were significantly higher than those of the control group, indicating that this nursing intervention model can improve the quality of life of patients with breast cancer. This may be related to the fact that we have made individual nursing programs for the breast cancer patients according to
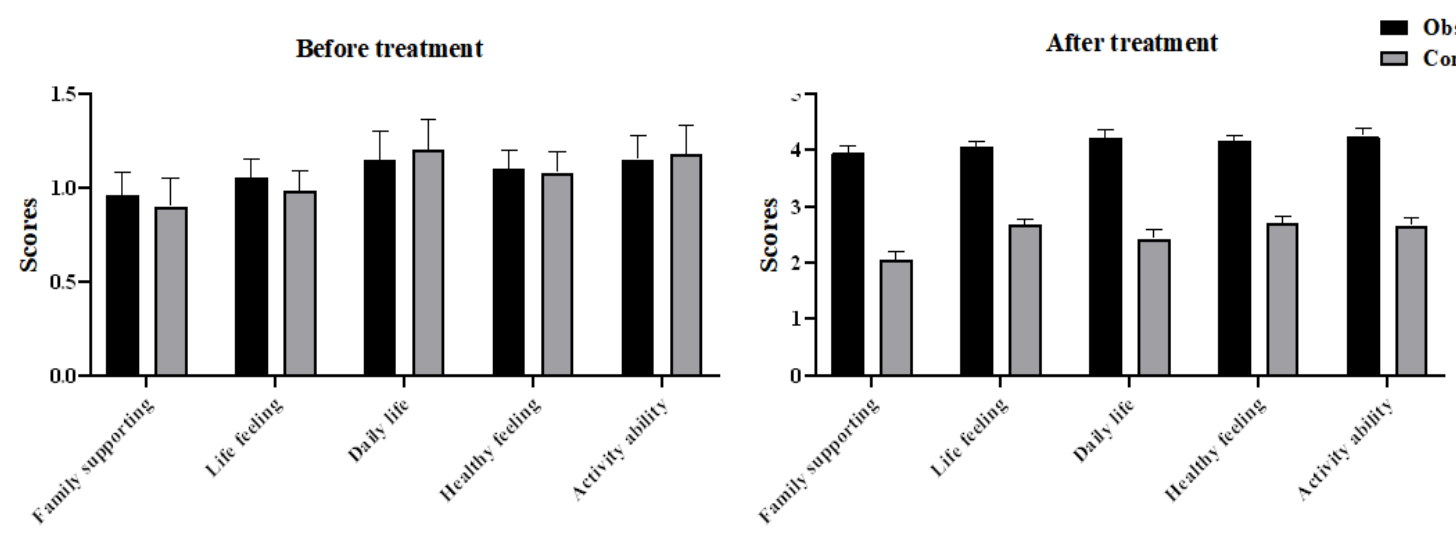

Fig. 3: Nursing management of the patients in two groups before and after treatment
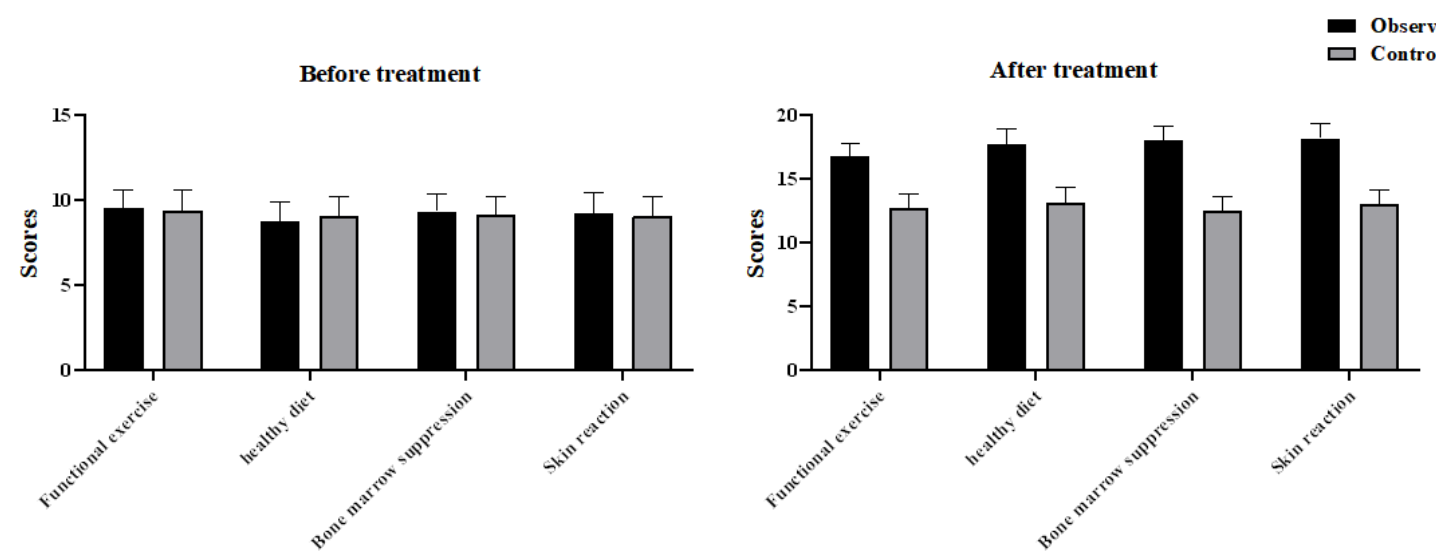

Fig. 4: Quality of life scores of patients in both groups before and after treatment 
PFS-R

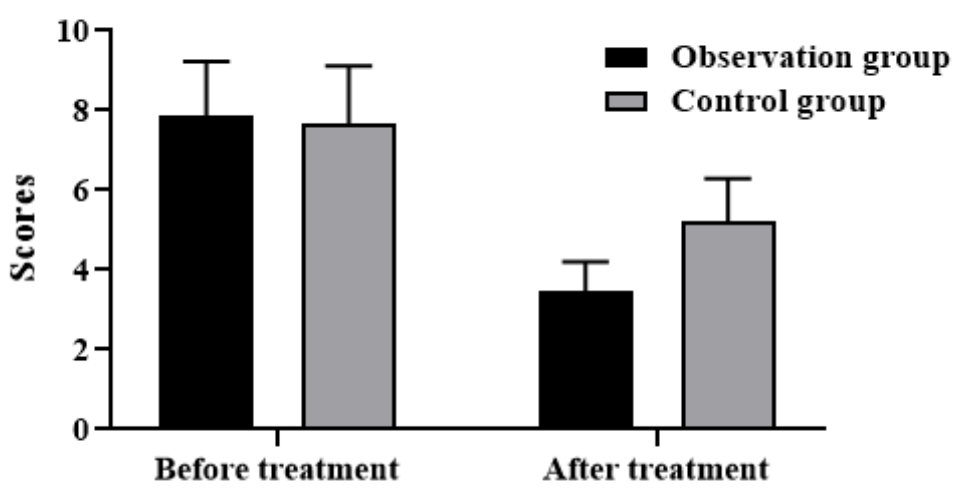

Fig. 5: PFS-R score of patients in two groups before and after treatment

different states in the nursing intervention model, and always grasp the patient's condition and psychological changes in the process of implementation, encourage the patients to actively cooperate with treatment, as far as possible to avoid the occurrence of adverse events during treatment. During the whole nursing period, we provided detailed answers to the patients in the observation group and their families, and obtained the changes of the patients' status through telephone follow-up twice a w, which played a positive role in stabilizing the patients' state during chemotherapy. In addition, this study also found that the compliance of treatment and the mastery of nursing health knowledge in the observation group were significantly higher than those in the control group, which may be related to our continuous guidance intervention on the patients in the observation group. Teach the patients about nursing health knowledge and some matters that need to be paid attention to in daily life, such as diet, activities, and medication before discharge. At the same time, patients were provided with nursing manuals and video and other materials commonly used in the hospital to help patients improve their interest in nursing learning and mastery of knowledge. Compared with routine nursing, continuous nursing guidance intervention pays more attention to details, such as medication, diet, exercise, and psychological intervention. Inform the patients of the difference and consequences between compliance and non-compliance, and inform the patients that diet and functional exercise can improve immunity and the effect of treatment effectively. In addition, patients were actively encouraged to participate in social activities, so that they can gain a sense of social identity and reduce the psychological burden of patients and the impact of negative emotions. In a 3-mo study of intervention care for breast cancer patients ${ }^{[18]}$, it was found that the continuous nursing intervention could improve the quality of life and mental state of patients significantly, and promote the therapeutic effect of postoperative radiotherapy and chemotherapy, which was consistent with our findings. Intervention participants reported less symptom burden compared with control participants. Therefore, the intervention can be recommended in its current form. It is also critical to examine the factors that contributed to the breast cancer and ensure to minimize them as the famous saying goes prevention is better than cure. To sum up, in the nursing intervention of breast cancer patients, the continuous intervention model can improve the life and mental state of breast cancer patients significantly, to improve their quality of life, alleviate the symptoms of cancer-related fatigue, and promote the rehabilitation of breast cancer patients.

\section{Acknowledgements:}

This work was supported by the Project of Jiangsu Science and Technology (BE2019757) and the Project of Nanjing Science and Technology (201803049).

\section{Conflicts of interest:}

The authors report no conflicts of interest.

\section{REFERENCES}

1. Frambes D, Lehto R, Sikorskii A, Tesnjak I, Given B, Wyatt G. Fidelity scorecard: evaluation of a caregiver-delivered symptom management intervention. J Adv Nurs 2017; 73:201221.

2. Heidrich SM, Brown RL, Egan JJ, Perez OA, Phelan $\mathrm{CH}$, Yeom $\mathrm{H}$, et al. An Individualized Representational Intervention to Improve Symptom Management (IRIS) in Older Breast Cancer Survivors: Three Pilot Studies. Oncol Nurs Forum 2009;36:E133.

3. Allard NC. Day Surgery for Breast Cancer: Effects of a Psychoeducational Telephone Intervention on Functional Status and Emotional Distress. Oncol Nurs Forum 2007;34.

4. Jennings-Sanders A, Kuo YF, Anderson ET, Freeman JL, Goodwin JS. How Do Nurse Case Managers Care for Older Women With Breast Cancer?. Oncol Nurs Forum 2005;32.

5. Allard N. Day surgery and recovery in women with a 
suspicious breast lesion: evaluation of a psychoeducational nursing intervention. Can Oncol Nurs J 2006;16:137-44.

6. Wang X, Lai Q, Tian Y, Zou L. Effect of evidence-based nursing intervention on upper limb function in postoperative radiotherapy patients with breast cancer. Medicine 2020;99:e19183.

7. Shu-Fang Z, Hospital T P. Analysis of the Effect of Nursing Intervention on the Quality of Life of Breast Cancer Patients. Systems Medicine 2019.

8. Rew L, Cauvin S, Cengiz A, Pretorius K, Johnson K. Application of project management tools and techniques to support nursing intervention research. Nursing Outlook 2020.

9. Hassan H E, El-Kholy G A, Ateya A A, Hassan A A. Breast Engorgement among Women with Caesarean Section: Impact of Nursing Intervention. Scholink 2020.

10. Lu Q, Dong L, Wu IH, You J, Huang J, Hu Y. The impact of an expressive writing intervention on quality of life among Chinese breast cancer patients undergoing chemotherapy. Supportive Care in Cancer 2019;27:165-73.

11. Jonczyk M, Jean J, Graham R, Chatterjee A. 3156 Breast Cancer Surgical Management: Novel Surgical Trends, Appropriate Axillary intervention, and associated Complications. J Clin Transl Sci 2019;3:120-1.

12. Xue-Lan H E, Ya-Qing C, Surgery D O. Application Effect of Cognitive Behavioral Nursing Intervention in Perioperative Period of Breast Cancer. Systems medicine 2019.

13. Junlan W. Effect of Buzhong Yiqi Decoction Combined with Nursing Intervention on Postoperative Cancer-induced Fatigue in Breast Cancer. Guangming J Chin Med 2019.
14. Hui X. Effect Observation of Early Nursing Intervention on Postoperative Limbs Functional Exercise of Breast Cancer patients. Smart Healthcare 2019.

15. Lina S, Liping Z. Effects of nursing intervention on life quality and joint function of patients with breast cancer after intensity modulated radiation therapy. Heb Med J 2019.

16. Hua-Ying T U. Analysis of the Influence of Operating Room Nursing Intervention on Stress Response in Patients with Breast Cancer Surgery. China \& Foreign Medical Treatment 2019.

17. Yali S U, Pilin W, June L, Jing H, Lin W. Effects of early comprehensive nursing intervention on hope and quality of life of breast cancer patients. Chinese Nursing Management 2019;19:310-3.

18. Hongyan W. Effect of Nursing Intervention Based on Maslow's Hierarchy of Needs Theory for Rehabilitation of Breast Cancer Patients undergoing Modified Radical Mastectomy. Medical innovation of china 2019.

This is an open access article distributed under the terms of the Creative Commons Attribution-NonCommercial-ShareAlike 3.0 License, which allows others to remix, tweak, and build upon the work non-commercially, as long as the author is credited and the new creations are licensed under the identical terms

This article was originally published in a special issue,
"Biomedical Research in Clinical and Preclinical
Pharmaceutics" Indian J Pharm Sci 2020:82(5)
Spl issue7;117-121

